\title{
TRANSVECTIONS IN SUBGROUPS OF THE GENERAL LINEAR GROUP CONTAINING A NONSPLIT MAXIMAL TORUS
}

\author{
V. A. KOIBAEV
}

\begin{abstract}
The objects of the study are intermediate subgroups of the general linear group $\operatorname{GL}(n, k)$ of degree $n$ over an arbitrary field $k$ that contain a nonsplit maximal torus associated with an extension of degree $n$ of the ground field $k$ (minisotropic torus). It is proved that if an overgroup of a nonsplit torus contains a one-dimensional transformation, then it contains an elementary transvection at some position in every column, and similarly for rows. This result makes it possible to associate net subgroups with groups of the above class and thus forms a base for their further study. This step is motivated by extremely high complexity of the lattice of intermediate subgroups. For a finite field, the lattice of overgroups of a nonsplit maximal torus is essentially determined by subfields intermediate between the ground field and its extension (G. M. Seitz, W. Kantor, R. Dye). Nothing like that holds true for an infinite field; even for the group $\operatorname{GL}(2, k)$ this lattice has much more complicated structure and essentially depends on the arithmetic of the ground field $k$ (Z. I. Borewicz, V. P. Platonov, Chan Ngoc Hoi, the author, and others).
\end{abstract}

\section{§1. INTRODUCTION}

Through the last decades, a lot of attention has been paid to the study of subgroups of classical and Chevalley groups that contain a split maximal torus. For the case of a split maximal torus, there is an almost complete description of the lattice of its overgroups in classical groups, and in Chevalley groups. The overgroups of split maximal tori were studied by A. Borel, J. Tits, G. M. Seitz, O. King, and others. We mention the important contribution of the Leningrad and St.-Petersburg algebra school (Z. I. Borewicz, N. A. Vavilov, and their students) to the description of overgroups of diagonal and block diagonal subgroups, over fields and rings. In the surveys [5, 6, 7, 8, and [27] one can find detailed descriptions of results in this direction.

Even for the general linear group over a field, much less is known about the description of overgroups of nonsplit maximal tori associated with a ground field extension (minisotropic tori). In a more general setting, this problem (related to the description of overgroups of subgroups from the Aschbacher class $C_{3}$ ) can be stated as follows. Let $K / k$ be a finite extension of degree $m$, let $V$ be a vector space of dimension $n$ over the field $K$ (and thus, of dimension $m n$ over $k$ ). Then, obviously (a $K$-linear map is $k$-linear), we have $\mathrm{GL}_{K}(V) \leq \mathrm{GL}_{k}(V)$, or, in the matrix form, $\mathrm{GL}(n, K) \leq \mathrm{GL}(m n, k)$. Observe that, for $n=1$, the group $\mathrm{GL}(1, K)=K^{*}$ is a nonsplit maximal torus. We state a result due to Shang Zhi Li 23], which reduces the above problem to the special case of a nonsplit torus. If $n \geq 3$, then for each intermediate subgroup $H$, SL $(n, K) \leq H \leq \mathrm{GL}(m n, k)=G$, there exists a unique intermediate subfield $k \leq L \leq K,[K: L]=d$ such that the group $H$ contains the group $\mathrm{SL}(d n, L)$ and is contained in its normalizer in $G$. In the case where

2010 Mathematics Subject Classification. Primary 20G15.

Key words and phrases. Overgroups, intermediate subgroups, nonsplit tori, maximal torus, transvection. 
$n=2, k \neq \mathbb{F}_{2}$, there is another series of intermediate subgroups, namely, the groups $\operatorname{Sp}(2 d, L)$ for all intermediate subfields $k \leq L \leq K,[K: L]=d$.

Observe that, for a finite field, Roger Dye (see [18, 19, 20]) described the overgroups of $\mathrm{GL}(n, K)$ in $\mathrm{GL}(n m, k)$ under the assumption $n \geq 1$.

As of today, a complete description of overgroups of a nonsplit maximal torus is only known in some special cases such as finite fields, or local fields. For finite fields, this was done in the work of Kantor, Seitz, and Dye (see [18]-[21], 25, 26]), where such a description was obtained for all fields of characteristic not equal to 2 or 3 that contain at least 13 elements. Observe that, in fact, Seitz described the subgroups of finite groups of Lie type containing an arbitrary maximal torus. Krupetskiı [14, 15] considered overgroups of some special tori (analogs of compact tori) in the unitary group defined over a nonramified quadratic extension of a local field (classification of such tori was addressed in 22]). For local and global fields, important results on overgroups of nonsplit tori were obtained by Platonov [24]. In the case of the field of real numbers $\mathbb{R}$, overgroups of maximal tori are closed in the usual real topology, and in particular, for a given torus there are only finitely many intermediate subgroups [17, 24].

As opposed to the case of finite fields, for infinite fields, overgroups of a nonsplit maximal torus correspond to a class of subrings of the ground field. Namely, the smallest subring $R_{0}$ of the ground field $k$ can be constructed such that every overgroup containing a nonsplit torus is associated in a natural sense with an elementary net subgroup, defined in terms of a net of ideals in some intermediate subring $R, R_{0} \leq R \leq k$.

As an example, we recall what the ring $R_{0}$ looks like in the case of $\operatorname{GL}(2, k)$; see [1]-4], 9]-[13, 16]. If $k$ is a local number field (a finite extension of the field of $p$-adic numbers, $p \neq 2$ ), and if the torus is determined by a nonramified quadratic extension of $k$, then the ring $R_{0}$ coincides with the ring of integers of the normalized valuation of $k$; in this case, there are countably many intermediate subgroups. For global fields the picture is much more complicated. When $k=\mathbb{Q}$ is the field of rational numbers, and the torus is determined by a quadratic extension $\mathbb{Q}(\sqrt{d})$, where $d \in \mathbb{Q}$ is square-free, then the ring $R_{0}$ consists of all rational numbers whose denominators contain only those primes modulo which $d$ is a quadratic residue. Thus, in this case, there is a continuum of overgroups of a nonsplit maximal torus in the group $\mathrm{GL}(2, \mathbb{Q})$. If $k=\mathbb{F}_{q}(t)$ is the field of rational functions with coefficients in a finite field $\mathbb{F}_{q}$ of odd characteristic, and if the torus is determined by a quadratic extension $k(\sqrt{\mu})$, where $\mu$ is a nonsquare in $\mathbb{F}_{q}$, then the ring $R_{0}$ coincides with the ring of all semiproper fractions $f / g, \operatorname{deg}(f) \leq \operatorname{deg}(g)$, whose denominators are products of irreducible polynomials of even degree over the field $\mathbb{F}_{q}$; again, there is a continuum of intermediate subgroups in this case.

The above results show that one should start with developing the concepts and terminology in which it would be possible to describe overgroups of nonsplit tori. It seems to us that, apart from congruences (nets), these concepts should come from geometry, and for specific fields also from their arithmetics.

A complete description of overgroups of a nonsplit maximal torus is difficult. As a first step, it is natural to consider such overgroups containing a one-dimensional transformation. This is precisely what we address in the present paper. We fix a base of the extension $K / k$ and prove that every subgroup of the above class contains an elementary transvection at some position in each column and in each row. This result allows us to construct net subgroups associated with overgroups of tori and thus creates a base for their further study.

Now we state the main results of the present paper. Let $K$ be a finite extension of degree $n$ of a field $k$ of odd characteristic. Next, let $T=T(K / k)$ be a nonsplit maximal torus corresponding to the extension $K / k$. 
Theorem 1. Let $H$ be an intermediate subgroup, $T \leq H \leq G=\mathrm{GL}(n, k)$, containing a one-dimensional transformation. Then for any $i, j$ there exist $r, s$ such that the group $H$ contains the elementary transvections $t_{i r}(\xi), t_{s j}(\zeta)$ for some nonzero elements $\xi, \zeta \in k$.

Theorem 2. Let $K=k(\sqrt[n]{d})$ be a radical extension of degree $n$ of a field $k$ of odd characteristic, and let $H$ be an intermediate subgroup, $T \leq H \leq G$, containing a onedimensional transformation. Then for any $i, j$, the group $H$ contains the elementary transvection $t_{i j}(\xi)$ for some nonzero $\xi \in k$.

In the present paper we use the following notation:

$E$ is the identity matrix of degree $n$;

$E_{i j}$ is the matrix that has $1 \in k$ at the position $(i, j)$ and zeros elsewhere;

$t_{i j}(\xi)=E+\xi E_{i j}$ is the elementary transvection, $\xi \in k^{*}, i \neq j$;

$\varepsilon_{i}=(0, \ldots, 1, \ldots, 0)$ is the vector that has 1 as its $i$ th component, whereas all other components are zeros;

by $[S]_{i j}$ we denote the entry of a matrix $S$ at the position $(i, j)$;

$S^{T}$ is the transpose of $S$;

a transformation $\tau$ of a vector space is said to be one-dimensional if $\operatorname{dim} \operatorname{Im}(\tau-1)=1$.

\section{§2. Matrix Representation of a nOnsplit maximal torus}

Let $K$ be an extension of degree $n$ of the field $k$. We fix a basis $e_{1}=1, e_{2}, \ldots, e_{n}$ of $K$ over $k$. Consider the regular embedding of the multiplicative group $K^{*}$ of the field $K$ in the group of $k$-linear automorphisms

$$
\underset{t \rightarrow \widehat{t}}{\longrightarrow} \operatorname{Aut}_{k}(K), \quad \widehat{t}(x)=t x, \quad x \in K .
$$

The image of $K^{*}$ under this embedding is denoted by $T=T(K / k)$ and is called the nonsplit maximal torus corresponding to the extension $K / k$. From the viewpoint of algebraic groups, this is an example of a minisotropic maximal torus.

In the above base, an element $t=x_{1} e_{1}+\cdots+x_{n} e_{n} \in K$ is represented by the matrix $C(t)=C(x), x=\left(x_{1}, \ldots, x_{n}\right)^{T}$,

$$
C(x)=\left(\begin{array}{cccc}
x_{1} & f_{12}(x) & \ldots & f_{1 n}(x) \\
x_{2} & f_{22}(x) & \ldots & f_{2 n}(x) \\
\ldots & \ldots & \ldots & \ldots \\
x_{n} & f_{n 2}(x) & \ldots & f_{n n}(x)
\end{array}\right) .
$$

Its first column coincides with $x$, while

$$
t \cdot e_{i}=f_{1 i}(x) e_{1}+\cdots+f_{n i}(x) e_{n}
$$

where $f_{i j}(x)=f_{i j}\left(x_{1}, \ldots, x_{n}\right)$ are the linear functionals $f_{i j}: k^{n} \longrightarrow k$ determined by the choice of the basis. Thus, the torus $T$ can be represented by the subgroup

$$
T=\left\{C(x): x \in k^{n} \backslash 0\right\}
$$

of the general linear group $G=\mathrm{GL}(n, k)$.

Let $t=x_{1} e_{1}+\cdots+x_{n} e_{n}, t^{-1}=x_{1}^{\prime} e_{1}+\cdots+x_{n}^{\prime} e_{n}, s=y_{1} e_{1}+\cdots+y_{n} e_{n}, s \cdot t=$ $z_{1} e_{1}+\cdots+z_{n} e_{n}$, and let $y=\left(y_{1}, \ldots, y_{n}\right)^{T}, z=\left(z_{1}, \ldots, z_{n}\right)^{T}, x^{\prime}=\left(x_{1}^{\prime}, \ldots, x_{n}^{\prime}\right)^{T}$.

Proposition 1. We have

$$
\begin{gathered}
C(x) C(y)=C(z), \quad z=C(x) y=C(y) x, \quad C\left(x^{\prime}\right)=C^{-1}(x), \\
C^{-1}(x) y=C(y) x^{\prime}, \quad C(C(x) y)=C(y) C(x) .
\end{gathered}
$$

Proof. The above formulas are implied by the fact that the embedding of $K^{*}$ in $\operatorname{Aut}_{k} K$ is regular. Observe that the last formula follows from the identity $\widehat{\widehat{t}(s)}=\widehat{t} \widehat{s}$. 
We note that the matrix $C(x)$ is scalar if and only if it represents an element of the ground field $k$. Next, for any $x \in k^{n} \backslash 0$ all columns and rows of the matrix $C(x)$ are nonzero, because otherwise the determinant of the matrix $C(x)$ equals zero. But this is impossible: it is equal to the norm of a nonzero element of the field $K$.

Proposition 2. 1) For any $\alpha, \beta \in k^{n} \backslash \overline{0}$ there exists $x \in k^{n} \backslash \overline{0}$ such that $C(x) \alpha=\beta$.

2) Let $\alpha \in k^{n}$ be an arbitrary column (row); then for any $i, 1 \leq i \leq n$, there exists $x \in k^{n}$ such that the ith row (column) of the matrix $C(x)$ coincides with $\alpha$.

Proof. The proof of statement 1) follows from the transitivity of the action of $K^{*}$ on $K^{*}$ by multiplication. To check statement 2), let $\alpha=\left(\alpha_{1}, \ldots, \alpha_{n}\right)$ be an arbitrary row. Set $t=\alpha_{1} e_{1}+\cdots+\alpha_{n} e_{n}$. Next, let $t e_{i}^{-1}=x_{1} e_{1}+\cdots+x_{n} e_{n}$. Then the $i$ th column of the matrix $C(x)$ coincides with the column $\alpha^{T}$. Indeed,

$$
\left(t e_{i}^{-1}\right) e_{i}=\alpha_{1} e_{1}+\cdots+\alpha_{n} e_{n} .
$$

Now, we show that the $i$ th row of some matrix $C(x)$ coincides with $\alpha$. Consider the $i$ th row of the matrix $C(x)$,

$$
f_{i 1}(x), f_{i 2}(x), \ldots, f_{i n}(x)
$$

where $f_{i j}(x)=a_{1 j}^{i} x_{1}+\cdots+a_{n j}^{i} x_{n}$ are the above linear functionals. The system of linear equations $f_{i j}(x)=a_{1 j}^{i} x_{1}+\cdots+a_{n j}^{i} x_{n}=\alpha_{j}, j=1, \ldots, n$, has a solution. Indeed, the determinant of the matrix $\left(a_{r s}^{i}\right)$ is not zero, because otherwise the corresponding homogeneous system has a nonzero solution $x \neq \overline{0}$ for which the matrix $C(x)$ has a zero row.

Proposition 3. Suppose a subgroup $H, T \leq H \leq G$, contains a transvection $\left(\delta_{i j}+\alpha_{i} \beta_{j}\right)$, $\sum_{i=1}^{n} \alpha_{i} \beta_{i}=0$. Then for every $i, 1 \leq i \leq n$, there exists a nonzero vector $\gamma=\left(\gamma_{1}, \ldots, \gamma_{n}\right)$ such that $\gamma_{i}=0$ and the transvection

$$
E+\gamma^{T} \cdot \varepsilon_{i}
$$

is contained in H. A similar claim holds true for transvections of the form $E+\varepsilon_{i}^{T} \cdot \gamma$.

Proof. Let $\alpha=\left(\alpha_{1}, \ldots, \alpha_{n}\right), \beta=\left(\beta_{1}, \ldots, \beta_{n}\right)$. By assumption, $E+\alpha^{T} \beta \in H$. Since $\beta \neq \overline{0}$, by Proposition 2 there exists $x \neq \overline{0}$ such that $\beta C(x)=\varepsilon_{i}$. Set $C^{-1}(x) \alpha^{T}=\gamma^{T}$. Then

$$
C^{-1}(x)\left(E+\alpha^{T} \beta\right) C(x)=E+\gamma^{T} \varepsilon_{i}
$$

Proposition 4. If all nondiagonal entries in some row or some column of a matrix $C(x)$ are zeros, then $C(x)$ is itself scalar.

Proof. First, observe that for $i \neq j$ the linear functional $f_{i j}(x)$ does not involve the variable $x_{1}$. This follows from the fact that

$$
C(x)=x_{1} E+x_{2} C\left(e_{2}\right)+\cdots+x_{n} C\left(e_{n}\right),
$$

where $C\left(e_{i}\right)$ is the matrix of the element $e_{i}$ (or, in other words, of the map $\widehat{e}_{i}$ ) in the basis $1, e_{2}, \ldots, e_{n}$.

Suppose, for instance, that all entries in the first row of the matrix $C(x)$, apart from the first one, are zeros, $f_{11}(x)=x_{1}, f_{1 j}(x)=0,2 \leq j \leq n$. By the above, the determinant of the homogeneous system $f_{11}(x)=x_{1}=0, f_{1 j}(x)=0,2 \leq j \leq n$, is nonzero and coincides with the determinant of the system $f_{1 j}(x)=0,2 \leq j \leq n$. Then this system has only the trivial solution $x_{2}=0, \ldots, x_{n}=0$, whence $C(x)=x_{1} E$. 


\section{§3. Bilinear FORM IndUCED By a nOnsplit TORUs, AND THE CORRESPONDING MATRICES}

It turns out that an important role in the study of subgroups containing a nonsplit torus is played by certain bilinear forms defined in terms of columns and rows of the matrices $C(x)$. Namely, for the $i$ th column (row) we define the bilinear form on $k^{n}$ as the entry of $C(x) C(y)$ at the position $(i, i)$,

$$
(x, y)^{i}=[C(x) C(y)]_{i i} .
$$

Usually, we work with the first column, so that we consider the bilinear form

$$
(x, y)=(x, y)^{1}=[C(x) C(y)]_{11}=x_{1} y_{1}+f_{12}(x) y_{2}+\cdots+f_{1 n}(x) y_{n},
$$

where $x, y \in k^{n}$. Since $C(x) C(y)=C(y) C(x)$, we have

$$
(x, y)=(y, x)=y_{1} x_{1}+f_{12}(y) x_{2}+\cdots+f_{1 n}(y) x_{n} .
$$

Consider the transvection $A=E+\alpha \cdot \varepsilon_{1}$,

$$
A=\left(\begin{array}{ccccc}
1 & 0 & 0 & \ldots & 0 \\
\alpha_{2} & 1 & 0 & \ldots & 0 \\
\alpha_{3} & 0 & 1 & \ldots & 0 \\
\ldots & \ldots & \ldots & \ldots & \ldots \\
\alpha_{n} & 0 & 0 & \ldots & 1
\end{array}\right)=E+\alpha \cdot \varepsilon_{1},
$$

where $\alpha=\left(0, \alpha_{2}, \ldots, \alpha_{n}\right)^{T}$.

Proposition 5. Let $C^{-1}(x)=C\left(x^{\prime}\right)$. Set

$$
S=S(x)=\left(C^{-1}(x)-\left(x^{\prime}, \alpha\right) E\right) A C(x) .
$$

Then $[S]_{1 i}=\delta_{1 i}$ (the Kronecker symbol).

Proof. It suffices to show that

$$
\left[C^{-1}(x) A^{-1}\left(C^{-1}(x)-\left(x^{\prime}, \alpha\right) E\right)^{-1}\right]_{1 i}=\delta_{1 i} .
$$

Indeed, the first row of the matrix $C^{-1}(x) A^{-1}$ has the form

$$
\left(x_{1}^{\prime}-\left(x^{\prime}, \alpha\right), f_{12}\left(x^{\prime}\right), \ldots, f_{1 n}\left(x^{\prime}\right)\right) .
$$

Thus, it coincides with the first row of the matrix $C^{-1}(x)-\left(x^{\prime}, \alpha\right) E$.

Proposition 6. We have

$$
[S, A]=S A S^{-1} A^{-1}=E+\gamma \cdot \varepsilon_{1},
$$

where $\gamma=\left(0, \gamma_{2}, \ldots, \gamma_{n}\right)^{T}$. Furthermore,

$$
\gamma=(S-E) \alpha
$$

Next,

$$
\gamma=\left[(x, \alpha) C^{-1}(x)-\left(x^{\prime}, \alpha\right) C(x)-(x, \alpha)\left(x^{\prime}, \alpha\right)\right] \alpha .
$$

Proof. Clearly, formula (2) follows from formula (1). Now, set $C=C(x), C^{-1}=C^{-1}(x)$. Then (1) implies that

$$
S-E=C^{-1} \alpha C-\left(x^{\prime}, \alpha\right) C-\left(x^{\prime}, \alpha\right) \alpha C .
$$

Thus,

$$
(S-E) \alpha=C^{-1} \alpha C \alpha-\left(x^{\prime}, \alpha\right) C \alpha-\left(x^{\prime}, \alpha\right) \alpha C \alpha .
$$

It only remains to observe that $\alpha C \alpha=(x, \alpha) \alpha$. 
Proposition 7. Assume that the group $H, T \leq H \leq G$, contains a transvection $A=$ $E+\alpha \varepsilon_{1}$. Then $H$ contains the transvections $E+\gamma \varepsilon_{1}, E+\gamma_{1} \varepsilon_{1}, E+\gamma_{2} \varepsilon_{1}$, where $\gamma$ is determined by (3),

$$
\gamma_{1}=2\left((x, \alpha) C^{-1}(x)-\left(x^{\prime}, \alpha\right) C(x)\right) \alpha, \quad \gamma_{2}=2(x, \alpha)\left(x^{\prime}, \alpha\right) \alpha .
$$

Proof. Since $E-\alpha \varepsilon_{1}=\left(E+\alpha \varepsilon_{1}\right)^{-1}$, we have $E+\alpha \varepsilon_{1}, E-\alpha \varepsilon_{1} \in H$. The relation $E+\gamma \varepsilon_{1} \in H$ follows from (3) and the fact that $H \geq T$. Since $E-\alpha \varepsilon_{1} \in H$, we can set $\alpha \equiv-\alpha$ in (3). Then we see that $E+\gamma_{3} \varepsilon_{1} \in H$, where

$$
\gamma_{3}=\left[(x, \alpha) C^{-1}(x)-\left(x^{\prime}, \alpha\right) C(x)+\left(x^{\prime}, \alpha\right)(x, \alpha)\right] \alpha .
$$

It remains to recall that $\left(E+\gamma \varepsilon_{1}\right)\left(E \pm \gamma_{3} \varepsilon_{1}\right)=E+\left(\gamma \pm \gamma_{3}\right) \varepsilon_{1} \in H$.

Observe that if $(K: k) \geq 3$, then there exists an element $\alpha \in K$ not contained in a quadratic extension of the field $k$. Indeed, let $x \in K$ be such that $\langle x, k\rangle$ is a quadratic extension of the field $k$, and let $y \in K \backslash\langle x, k\rangle$ be such that $\langle y, k\rangle$ is a quadratic extension of the field $k$. Then $\alpha=x+y$ is not contained in a quadratic extension of the field $k$.

A fundamental role in further arguments is played by the matrices occurring in (4).

Lemma 1. Let $n \geq 3$. Set

$$
B(x)=(x, \alpha) C^{-1}(x)-\left(x^{\prime}, \alpha\right) C(x),
$$

where $\alpha=\left(0, \alpha_{2}, \ldots, \alpha_{n}\right)^{T}$ is a vector with at least two nonzero components. Then for some $x$ the matrix $B(x)$ is not scalar.

Proof. Let $\alpha_{i}, \alpha_{j} \neq 0, i \neq j, i \geq 2, j \geq 2$. We choose a vector $y$ so that the first row of the matrix $C(y)$ has 1 at the $i$ th position and zeros elsewhere. This is possible by Proposition 2. Similarly, let $z$ be a vector such that the first row of the matrix $C(z)$ has 1 at the $j$ th position and zeros elsewhere. If one of the matrices $B(y)$ or $B(z)$ is not scalar, we are done. Thus, we may assume that both of them are scalar,

$$
\begin{aligned}
& B(y)=(y, \alpha) C^{-1}(y)-\left(y^{\prime}, \alpha\right) C(y)=\xi_{1} E, \\
& B(z)=(z, \alpha) C^{-1}(z)-\left(z^{\prime}, \alpha\right) C(z)=\xi_{2} E,
\end{aligned}
$$

where $\xi_{1}, \xi_{2} \in k$. By the choice of $y$ and $z$, we have

$$
(y, \alpha)=\alpha_{i} \neq 0, \quad(z, \alpha)=\alpha_{j} \neq 0 .
$$

Observe that the matrices $C(y)$ and $C(z)$ are not scalar, and

$$
C(y) \neq \zeta C(z)+\zeta_{1} E
$$

for all $\zeta, \zeta_{1} \in k$. Next, observe that $\left(y^{\prime}, \alpha\right) \neq 0,\left(z^{\prime}, \alpha\right) \neq 0$, because otherwise from (5) and (6) it would follow that one of the matrices $C(y)$ or $C(z)$ is scalar. Now, (5) and (7) imply that $\langle C(z), k\rangle$ and $\langle C(y), k\rangle$ are quadratic extensions of the field $k$, and, moreover, $C(z) \in K \backslash\langle C(y), k\rangle$.

We choose $\xi_{0} \in k$ in such a way that $\left(y+\xi_{0} z, \alpha\right) \neq 0$. For this, observe that

$$
\left(y+\xi_{0} z, \alpha\right)=(y, \alpha)+\xi_{0}(z, \alpha)=\alpha_{i}+\xi_{0} \alpha_{j} .
$$

Set $\xi_{0} \neq-\frac{\alpha_{i}}{\alpha_{j}}, \xi_{0} \neq 0$. Now, let $x=y+\xi_{0} z$. Then $(x, \alpha) \neq 0$. We show that the matrix

$$
B(x)=(\alpha, x) C^{-1}(x)-\left(x^{\prime}, \alpha\right) C(x)
$$

is not scalar. Suppose $B(x)$ is scalar, contrary to expectations. Let

$$
(\alpha, x) C^{-1}(x)-\left(x^{\prime}, \alpha\right) C(x)=\zeta_{0} E .
$$

Observe that $\left(x^{\prime}, \alpha\right) \neq 0$, because otherwise $C(x)=C(y)+\xi_{0} C(z)$ is a scalar matrix, which contradicts $(7)$. Thus, $\langle C(x), k\rangle$ is a quadratic extension of the field $k$. It only remains to notice that $C(x)=C(y)+\xi_{0} C(z)$, and, as we have shown above, $\langle C(z), k\rangle$ 
and $\langle C(y), k\rangle$ are quadratic extensions of the field $k$ and $C(z) \in K \backslash\langle C(y), k\rangle$. This contradicts the arguments immediately preceding the lemma.

\section{§4. A SUBGROUP CONTAINING A ONE-DIMENSIONAL TRANSFORMATION CONTAINS A TRANSVECTION}

In the present section we show that if an intermediate subgroup $H, T \leq H \leq G$, contains a one-dimensional transformation, it already contains a transvection.

Let $A=\operatorname{diag}(\theta, 1, \ldots, 1)$ be a diagonal matrix, $\theta \neq 1, A=E+\alpha \varepsilon_{1}$, where $\alpha=$ $(\theta-1) \varepsilon_{1}^{T}$. Clearly, $A^{-1}=E-\frac{1}{\theta} \alpha \varepsilon_{1}$. Set

$$
S=\left(C^{-1}(x)-\frac{1}{\theta}\left(x^{\prime}, \alpha\right) E\right) A C(x),
$$

where $\left(x^{\prime}, \alpha\right)=x_{1}^{\prime}(\theta-1), x^{\prime}=\left(x_{1}^{\prime}, \ldots, x_{n}^{\prime}\right)^{T}, C^{-1}(x)=C\left(x^{\prime}\right), x=\left(x_{1}, \ldots, x_{n}\right)^{T}$.

Lemma 2. We have

$$
[S, A]=E+\gamma \varepsilon_{1},
$$

where

$$
\gamma=\frac{(\theta-1)^{2}}{\theta^{2}}\left(-x_{1}^{\prime} x+\theta x_{1} x^{\prime}-(\theta-1) x_{1}^{\prime} x_{1} \varepsilon_{1}^{T}\right) .
$$

Proof. We immediately see that $\gamma=\frac{1}{\theta}(S-E) \alpha$, whence

$$
\gamma=\frac{1}{\theta^{2}}\left[-\left(x^{\prime}, \alpha\right) C(x) \alpha+\theta C^{-1}(x) \alpha C(x) \alpha-\left(x^{\prime}, \alpha\right) \alpha C(x) \alpha\right] .
$$

This implies the lemma.

Remark 1 . It is easily seen that the matrix $E+\gamma \varepsilon_{1}$ from the above lemma is a transvection. For this it suffices to observe that $\gamma^{T}=\left(0, \gamma_{2}, \ldots, \gamma_{n}\right)$.

Proposition 8. If an intermediate subgroup $H, T \leq H \leq G$, contains a one-dimensional transformation, then $H$ contains a transvection.

Proof. First, observe that if in Lemma 2 we take a vector $x$ such that $x_{1}=0, x_{1}^{\prime} \neq 0$, then the above matrix $E+\gamma \varepsilon_{1}$ is a transvection. In particular, this implies that if the subgroup $H$ contains a diagonal matrix $\operatorname{diag}(\theta, 1, \ldots, 1)$, then it contains a transvection. Now, assume that $H, H \leq \operatorname{Aut}_{k}(K)$, contains a one-dimensional transformation $\tau: K \rightarrow K$ that is not a transvection. Then $\tau=1_{K}+\varphi \cdot \alpha, \alpha \in K, \varphi: K \rightarrow k$, where $1_{K}$ is the identity map. Since $\tau$ is not a transvection, we have $\varphi(\alpha) \neq 0$. Since $K^{*} \leq H$, the transformation

$$
\alpha^{-1}\left(1_{K}+\varphi \cdot \alpha\right) \alpha=1_{K}+(\varphi \circ \alpha) \cdot 1
$$

is contained in $H$. Thus, we may assume that $\tau=1_{K}+\varphi \cdot 1,1 \in K$, where $\varphi(1) \neq 0$.

We take a basis $e_{2}^{\prime}, \ldots, e_{n}^{\prime}$ of the subspace $\operatorname{ker} \varphi$. Recalling that $1 \notin \operatorname{ker} \varphi$, we see that $1=e_{1}^{\prime}, e_{2}^{\prime}, \ldots, e_{n}^{\prime}$ is a basis of $K$ over $k$. In this basis, the matrix of the transformation $\tau$ is diagonal, $\operatorname{diag}(\theta, 1, \ldots, 1), \theta=\varphi(1)$. Then $H$ contains a transvection by Lemma 2 . It suffices to return to the initial basis. Note that the transvections are invariant under a basis change.

\section{§5. Techniques of extraction of Elementary transvections}

In this section we develop the techniques of extraction of elementary transvections from intermediate subgroups $H, T \leq H \leq G$, containing a one-dimensional transformation. By $\S 4$, we may assume that the subgroup $H$ contains a transvection, not necessarily elementary. Next, by Proposition 3 we can pick an $A \in H$ of the form $A=E+\alpha \varepsilon_{1}$, where all entries of the matrix $A-E$ apart from the first column are zeros, whereas the first column of $A-E$ coincides with the column $\alpha$, where $\alpha^{T}=\left(0, \alpha_{2}, \ldots, \alpha_{n}\right)$. By 
Proposition 7 , together with the matrix $A$, for each $x \in k^{n} \backslash \overline{0}$, the subgroup $H$ contains a transvection $E+\gamma \varepsilon_{1}$, where

$$
\gamma=\left[(x, \alpha) C^{-1}(x)-\left(x^{\prime}, \alpha\right) C(x)\right] \alpha .
$$

Proposition 9. Suppose there exists $k, k \geq 2$, such that $C^{-1}(x) \alpha=\varepsilon_{k}^{T}$ and $(x, \alpha) \neq 0$. Then $t_{k 1}((x, \alpha)) \in H$.

Proof. By assumption, $\alpha$ coincides with the $k$ th column of the matrix $C(x)$. Consequently, $\alpha$ is orthogonal to the first row of the matrix $C^{-1}(x)$, whence $\left(x^{\prime}, \alpha\right)=0$. But then (8) implies that $\gamma=(x, \alpha) \varepsilon_{k}^{T}$, and finally, $E+\gamma \varepsilon_{1}=t_{k 1}((x, \alpha)) \in H$.

By Proposition 9, in the sequel we may assume that $\alpha$ is subject to the conditions of the following proposition.

Proposition 10. Suppose $\alpha=\left(0, \alpha_{2}, \ldots, \alpha_{n}\right)^{T}, \alpha_{1}=0, t=\alpha_{2} e_{2}+\cdots+\alpha_{n} e_{n} \in K$. The following conditions are equivalent:

(a) for each $k \geq 2$, if $\alpha$ is the $k$ th column of the matrix $C(x)$, then $(x, \alpha)=0$;

(b) $\left[C\left(t^{2} e_{k}^{-2}\right)\right]_{1 k}=0$ for each $k \geq 2$;

(c) for each $k \geq 2$, the element $t^{2} e_{k}^{-1}$ is contained in the $k$-subspace $\left\langle e_{2}, \ldots, e_{n}\right\rangle$ of dimension $n-1$.

Proof. Observe that

$$
\left(t e_{k}^{-1}\right) e_{k}=\alpha_{1} \cdot e_{1}+\alpha_{2} \cdot e_{2}+\cdots+\alpha_{n} e_{n} .
$$

Consequently, $C\left(t e_{k}^{-1}\right)$ is a matrix of the form $C(x)$, whose $k$ th column coincides with $\alpha$. Then condition $(x, \alpha)=0$ amounts to the orthogonality of the vector $\alpha$ to the first row of the matrix $C(x)$, and thus also to the first row of the matrix $C\left(t e_{k}^{-1}\right)$. Therefore, the relation $(x, \alpha)=0$ is equivalent to the orthogonality of the $k$ th column of the matrix $C\left(t e_{k}^{-1}\right)$ to its first row, i.e., $\left[C^{2}\left(t e_{k}^{-1}\right)\right]_{2 k}=\left[C\left(t^{2} e_{k}^{-2}\right)\right]_{1 k}=0$. This shows that conditions (a) and (b) are equivalent. The equivalence of (b) and (c) follows from the definition of the matrix $C\left(t^{2} e_{k}^{-2}\right)$.

Remark 2. Here and in what follows, $C(q)$ denotes the matrix of an element $q \in K$ (viewed as an element of $\left.\operatorname{Aut}_{k}(K)\right)$ in the basis $e_{1}, \ldots, e_{n}$.

Remark 3. The proof of Proposition 10 shows that the condition $t^{2} e_{k}^{-1} \notin\left\langle e_{2}, \ldots, e_{n}\right\rangle$ is equivalent to the fact that $t_{k 1}((x, \alpha)) \in H$ for some $x \in k^{n} \backslash \overline{0}$ with $(x, \alpha) \neq 0$.

Denote by $t_{B}$ the element of the field $K$ corresponding to the matrix $B(x) \in T$; see Lemma 1. Propositions 7, 9, 10, and the last remark can be summarized as follows.

Proposition 11. Suppose an intermediate subgroup $H$ contains a transvection $E+\alpha \varepsilon_{1}$, $\alpha^{T}=\left(0, \alpha_{2}, \ldots, \alpha_{n}\right), t=\alpha_{2} e_{2}+\cdots+\alpha_{n} e_{n} \in K, k \geq 2$. If at least one of the elements $t^{2} e_{k}^{-1}, t_{B} t^{2} e_{k}^{-1}, t_{B}^{2} t^{2} e_{k}^{-1}$ is not contained in the $k$-subspace $\left\langle e_{2}, \ldots, e_{n}\right\rangle$, then

$$
t_{k 1}(\xi) \in H
$$

for some $\xi \neq 0$.

\section{§6. Proof of the main Results}

In the present section we prove Theorems 1 and 2 .

First, we prove that it suffices to consider the transvection $A=E+\alpha \varepsilon_{1}$ corresponding to the first column $\alpha=\left(0, \alpha_{2}, \ldots, \alpha_{n}\right)^{T}$. Indeed, consider the transvection $A=E+\alpha \varepsilon_{i}$, $\alpha=\left(\alpha_{1}, \alpha_{2}, \ldots, \alpha_{n}\right)^{T}, \alpha_{i}=0$, with nonzero $i$ th column of the matrix $A-E$. If we consider the bilinear form $(x, y)=(x, y)^{i}=[C(x) C(y)]_{i i}$ for the $i$ th column, see $\S 3$, then, defining the matrix $S=S(x)$ by formula (1) in $\S 3$, we easily check that the claims 
of Proposition 6 and 7 hold true. Thus, to the matrix $A=E+\alpha \varepsilon_{i}$ we can apply all other results of $\S \S 3-5$.

Similarly, considering the action of elements of the torus on rows (the transposition of the above action on columns), we can reduce the case of transvections with nontrivial rows to that of transvections with nontrivial columns.

Proof of Theorem 1. Let $\tau \in H$ be a one-dimensional transformation $\tau=\left(\delta_{i j}+\alpha_{i} \beta_{j}\right)$. By Proposition 8, we may assume that $\tau$ is a transvection, in other words, that $\sum_{i=1}^{n} \alpha_{i} \beta_{i}=0$. Next, by Proposition 3 and the above, we may assume that

$$
\tau=A=E+\alpha \varepsilon_{1},
$$

where $\alpha=\left(0, \alpha_{2}, \ldots, \alpha_{n}\right)^{T}$.

We must show that for some $k \geq 2$ and $\zeta \neq 0$ the elementary transvection $t_{k 1}(\zeta)$ is contained in the group $H$.

We may assume that for our basis $e_{1}=1, e_{2}, \ldots, e_{n}$, the elements $e_{2}^{-1}, \ldots, e_{n}^{-1}$ are linearly independent over the field $k$. By Proposition 11, it suffices to show that, for some $k \geq 2$, either $t^{2} e_{k}^{-1}$ or $t_{B} t^{2} e_{k}^{-1}$ is not contained in the $k$-subspace $L=\left\langle e_{2}, \ldots, e_{n}\right\rangle$. Suppose this is not the case. Let $t^{2} e_{k}^{-1} \in L, t_{B} t^{2} e_{k}^{-1} \in L$ for all $k, 2 \leq k \leq n$. Lemma 1 allows us to assume that the element $t_{B}$ is not contained in the field $k$. Set $L_{1}=\left\langle e_{2}^{-1}, \ldots, e_{n}^{-1}\right\rangle$. Then $t^{2} L_{1}=L$, whence $t_{B} L=L$. But then the first row of the matrix $B=B(x)$ corresponding to the element $t_{B}$ in the basis $e_{1}=1, e_{2}, \ldots, e_{n}$, has the form $\left(b_{11}, 0, \ldots, 0\right)$. On the other hand, $B(x)=C(z)$, where $z=(x, \alpha) x^{\prime}-\left(x^{\prime}, \alpha\right) x$; compare Proposition 1 and Lemma 1. Proposition 4 implies that the matrix $C(z)$, and thus also the matrix $B(x)$, are scalar, whence $t_{B} \in k$. This contradicts the fact that the element $t_{B}$ is not contained in the field $k$.

Remark 4. In the proof we used the fact that the reciprocals $1 / e_{2}, \ldots, 1 / e_{n}$ of the basis elements are linearly independent.

Remark 5. Theorem 1 is valid without the assumption $e_{1}=1$.

Let $x^{n}-d$ be an irreducible polynomial over the field $k, d \in k$. Then the elements $e_{i}=\theta^{i-1}, 1 \leq i \leq n$, form a basis of the radical extension $K=k(\sqrt[n]{d}), \theta=\sqrt[n]{d}$, of the field $K=k(\theta)$ over $k$. We state another result, which will be used in the proof of Theorem 2 .

Proposition 12. Suppose $n \geq 3, i \neq s, 2 \leq s \leq n, 1 \leq i \leq n$. Then there exists $\omega=x_{1}+x_{2} \theta+\cdots+x_{n} \theta^{n-1} \in K$ such that $x_{i} \neq 0, x_{s}=0$, and $x_{s}^{\prime} \neq 0$, where $\omega^{-1}=x_{1}^{\prime}+x_{2}^{\prime} \theta+\cdots+x_{n}^{\prime} \theta^{n-1}$.

Proof. It is easy to verify that

$$
(1+\theta)^{-1}=\frac{1}{1+(-1)^{n-1} d}\left(1-\theta+\theta^{2}-\cdots+(-1)^{n-1} \theta^{n-1}\right) .
$$

It follows that, for any integer $j$,

$$
\left(\theta^{j}+\theta^{j+1}\right)^{-1}=\theta^{-j}(1+\theta)^{-1}=z_{1}+z_{2} \theta+\cdots+z_{n} \theta^{n-1},
$$

where $z_{i} \neq 0$ for all $i, 1 \leq i \leq n$. Now, the proof of the proposition can be finished as follows. If $2 \leq s<i \leq n$, then we can take $\omega=\theta^{i-1}+\theta^{i}$, obtaining $x_{s}=0, x_{i}=1$, $x_{s}^{\prime} \neq 0$. Let $n \geq s>i \geq 1$. If $i \geq 2$, then for $\omega=\theta^{i-2}+\theta^{i-1}$ we get $x_{i}=1, x_{s}=0$ and, by the above, $x_{s}^{\prime} \neq 0$. In the case where $i=1$ we do the following. For $s \geq 3$ we can take $\omega=1+\theta$ such that $x_{2}=x_{1}=1, x_{s}=0$, and $x_{s}^{\prime} \neq 0$. On the other hand, for $s=2$ we take the element $\omega=\theta^{-1}+1$, for which $x_{1}=1, x_{n}=1 / d, n \geq 3, n>s, x_{s}=0$, $x_{s}^{\prime} \neq 0$. 
Proof of Theorem 2. For $n=2$ our claim follows from Theorem 1. Thus, we assume that $n \geq 3$. By Theorem 1 it suffices to show that if $t_{k r}(\xi) \in H$ for some $k, r, \xi \neq 0$, then $t_{j r}\left(\xi_{1}\right) \in H$ for all $j, j \neq r, 1 \leq j \leq n$, and for some $\xi_{1} \neq 0$. Without loss of generality we may assume that $r=1, t_{k 1}(\xi) \in H, t_{k 1}(\xi)=E+\alpha^{T} \varepsilon_{1}$, where $\alpha=\xi \varepsilon_{k}, k \geq 2$. Then the vector $\alpha$ corresponds to an element $t=\theta^{k-1}$ of the field $K$, where for simplicity we omit the coefficient $\xi$. Since $2 \leq k \leq n$, we have $2 \leq n-k+2 \leq n$. Since $n \geq 3$, we can choose an index $i, 1 \leq i \leq n$, such that $i \neq n-k+2$. By Proposition 12, there is a vector $x=\left(x_{1}, \ldots, x_{n}\right)$ subject to the conditions $x_{i} \neq 0, x_{n-k+2}=0, x_{n-k+2}^{\prime} \neq 0$. The elements of the matrix $C(x)$ are calculated by the formulas

$$
[C(x)]_{i j}= \begin{cases}x_{i+1-j} & \text { if } j \leq i \\ d x_{n+i+1-j} & \text { if } j \geq i+1\end{cases}
$$

In particular, $[C(x)]_{1 k}=d x_{n-k+2}$, and for the inverse matrix $C^{-1}(x)=C\left(x^{\prime}\right)$ we have $\left[C^{-1}(x)\right]=d x_{n-k+2}^{\prime}$. For the above $x$, we have

$$
\left(x^{\prime}, \alpha\right)=d \xi x_{n-k+2}^{\prime} \neq 0, \quad(x, \alpha)=d \xi x_{n-k+2}=0 .
$$

Then the matrix $B(x)$ considered in $\S 3$ equals

$$
B(x)=(x, \alpha) C^{-1}(x)-\left(x^{\prime}, \alpha\right) C(x)=C(x),
$$

where the nonzero coefficient $-d \xi x_{n-k+2}^{\prime}$ is omitted.

The matrix $B(x)=C(x)$ corresponds to the element $t_{B}=\sum_{i=1}^{n} x_{i} \theta^{i-1}$, where $x_{n-k+2}=0$. Since $t=\theta^{k-1}$, it follows that

$$
t_{B} t^{2}=x_{1} \theta^{2(k-1)}+\cdots+x_{i} \theta^{2(k-1)+i-1}+\cdots+x_{n} \theta^{2(k-1)+n-1} .
$$

Observe that $i$ is arbitrary, $x_{i} \neq 0,1 \leq i \leq n$, and, moreover, $i \neq n-k+2$, $x_{n-k+2}=0$. Thus, the degree of $\theta$ at $x_{n-k+2}$ equals

$$
2(k-1)+n-k+2-1 \equiv(k-1)(\bmod n) .
$$

It follows that as $i$ ranges between 1 and $n, i \neq n-k+2$, the degrees $2(k-1)+i-1$ run over a complete residue system modulo $n$, with the exception of $(k-1)(\bmod n)$. Let $j$ be arbitrary, $1 \leq j \leq n-1, j \neq k-1, x_{i} \theta^{2(k-1)+i-1}=x_{i} \zeta \theta^{j}$ for some $\zeta \in k, \zeta \neq 0$. All other degrees of the element $\theta$ as in (9) run over all values from 0 to $n-1$, with the exception of $j$ and $k-1$.

Then

$$
t_{B} t^{2} \theta^{-j}=x_{i} \zeta+\zeta_{1} \theta+\cdots+\zeta_{n-1} \theta^{n-1}
$$

where $x_{i} \zeta \neq 0$. Thus, $t_{B} t^{2} \theta^{-j} \notin\left\langle\theta, \theta^{2}, \ldots, \theta^{n-1}\right\rangle$. Recall that for our basis we have $e_{j+1}=\theta^{j}$; hence, by Proposition $11, t_{j+1,1}\left(\xi_{1}\right) \in H, \xi_{1} \neq 0$, with an arbitrary $j$, $j \neq k-1$. But since $t_{k 1}(\xi) \in H$, for any $j$ with $2 \leq j \leq n$ there exists $\xi \neq 0$ such that $t_{j 1}(\xi) \in H$ (in general, with different $\xi$ 's for different $j$ 's).

The author thanks Nikolaŭ Vavilov who raised some important questions related to the present paper.

\section{REFERENCES}

[1] A. A. Bondarenko, The arrangement of subgroups that contain a nonramified quadratic torus in the general linear group of degree 2 over a local number field $(p \neq 2)$, Zap. Nauchn. Sem. S.-Peterburg. Otdel. Mat. Inst. Steklov. (POMI) 211 (1994), 67-79; English transl., J. Math. Sci. (New York) 83 (1997), no. 5, 600-608. MR.1333874 (96k:20091)

[2] _ The arrangement of subgroups that contain a nonramified quadratic torus in the general linear group of degree 2 over a local number field $(p=2)$, Zap. Nauchn. Sem. S.-Peterburg. Otdel. Mat. Inst. Steklov. (POMI) 211 (1994), 80-90; English transl., J. Math. Sci. (New York) 83 (1997), no. 5, 609-616. MR:1333875 (96k:20092) 
[3] Z. I. Borevich and V. A. Koŭbaev, On rings of multipliers associated with intermediate subgroups for a square torus, Vestnik S.-Peterburg. Univ. Mat. Mekh. Astronom. 1993, vyp. 2, 5-10; English transl., Vestnik St. Petersburg Univ. Math. 26 (1993), no. 2, 1-5. MR.1370225 (96k:20086)

[4] Z. I. Borevich, V. A. Koubaev, and Chan Ngok Khor, Lattices of subgroups in $\mathrm{GL}(2, \mathbb{Q})$ that contain a nonsplittable torus, Zap. Nauchn. Sem. Leningrad. Otdel. Mat. Inst. Steklov. (LOMI) 191 (1991), 24-43; English transl., J. Soviet Math. 63 (1993), no. 6, 622-633. MR.1112379 (92g:20068)

[5] N. A. Vavilov, Subgroups of splittable classical groups, Trudy Mat. Inst. Steklov. 183 (1990), 29-42; English transl., Proc. Steklov Inst. Math. 1991, no. 4, 27-41. MR.1092012 (92d:20065)

[6] Subgroups of Chevalley groups that contain a maximal torus, Trudy Leningrad. Mat. Obshch.1 (1990), 64-109; English transl., Amer. Math. Soc. Transl. Ser. 2, vol. 155, Amer. Math. Soc., Providence, RI, 1993, pp. 59-100. MR.1104207 (92f:20046)

[7] N. A. Vavilov and V. V. Nesterov, Geometry of microweight tori, Vladikavkaz. Mat. Zh. 10 (2008), no. 1, 10-23. (Russian) MR2434648 (2009g:20105)

[8] N. A. Vavilov and A. V. Stepanov, Overgroups of semisimple groups, Vestnik Samar. Gos. Univ. Estestvennonauchn. Ser. 2008, no. 3, 51-95. (Russian) MR2473730 (2009k:20113)

[9] V. S. Dzigoeva and V. A. Koĭbaev, Intermediate subgroups in the second-order general linear group over the field of rational functions containing a square torus, Vladikavkaz. Mat. Zh. 10 (2008), no. 1, 27-34. (Russian) MR2434650 (2010d:20058)

[10] N. A. Dzhusoeva and V. A. Korbaev, Maximal subgroups that contain a torus, which are connected with the field of fractions of a Dedekind domain, Zap. Nauchn. Sem. S.-Peterburg. Otdel. Mat. Inst. Steklov. (POMI) 289 (2002), 149-153; English transl., J. Math. Sci. (New York) 124 (2004), no. 1, 4763-4765. MR1949739 (2003k:20074)

[11] V. A. Kolbaev, Subgroups of the group $\mathrm{GL}(2, \mathbb{Q})$ that contain a nonsplittable maximal torus, Dokl. Akad. Nauk SSSR 312 (1990), no. 1, 36-38; English transl., Soviet Math. Dokl. 41 (1990), no. 3, 414-416 (1991). MR.1072859 (91j:20109)

$[12]$, Subgroups of the group $\operatorname{GL}(2, k)$ that contain a nonsplittable maximal torus, Zap. Nauchn. Sem. S.-Peterburg. Otdel. Mat. Inst. Steklov. (POMI) 211 (1994), 136-145; English transl., J. Math. Sci. (New York) 83 (1997), no. 5, 648-653. MR1333880 (96d:20044)

[13] __ Subgroups of a general linear group that contain a maximal unsplittable torus concerned with the radical extension, Vestnik S.-Peterburg. Univ. Mat. Mekh. Astronom. 1995, vyp. 1, 29-33; English transl., Vestnik St. Petersburg Univ. Math. 28 (1995), no. 1, 24-27. MR.1794675

[14] S. L. Krupetski1̌, Subgroups of the unitary group over a local field, Zap. Nauchn. Sem. Leningrad. Otdel. Mat. Inst. Steklov. (LOMI) 94 (1979), 81-103; English transl. in J. Soviet Math. 19 (1982), no. 1. MR0571518 (81j:20068)

[15] _ Subgroups of the unitary group over a 2-adic local field, Zap. Nauchn. Sem. Leningrad. Otdel. Mat. Inst. Steklov. (LOMI) 103 (1980), 79-89; English transl. in J. Soviet Math. (New York) 24 (1984), no. 4. MR0618499 (83a:20058)

[16] Chan Ngok Khou, Arrangement of subgroups in $\mathrm{GL}(2, \mathbb{Q})$ that contain a nonramified torus, Kandidate Diss., Leningrad. Gos. Univ., Leningrad, 1990, pp. 1-182. (Russian)

[17] D. Z. Djoković, Subgroups of compact Lie groups containing a maximal torus are closed, Proc. Amer. Math. Soc. 83 (1981), no. 2, 431-432. MR0624947 (83m:22014)

[18] R. H. Dye, Maximal subgroups of symplectic groups stabilizing spreads. I, II, J. Algebra 87 (1984), no. 2, 493-509; J. London Math. Soc. (2) 40 (1989), no. 2, 215-226. MR0739949 (85h:20054) MR 1044270 (91d:20053)

[19] - Maximal subgroups of $\operatorname{PSp}_{6 n}(q)$ stabilizing spreads of totally isotropic planes, J. Algebra 99 (1986), 191-209. MR0836642 (87d:20061)

[20] Ann. Mat. Pura Appl. (4) 158 (1991), 33-50. MR.1131844 (92k:20092)

[21] W. M. Kantor, Linear groups containing a Singer cycle, J. Algebra 62 (1980), no. 1, 232-234. MR0561126 (81g:20089)

[22] K. Kariyama, On conjugacy classes of maximal tori in classical groups, J. Algebra 125 (1989), no. 1, 133-149. MR1012667 (90j:20090)

[23] Shang Zhi Li, Overgroups in $\mathrm{GL}(n r, F)$ of certain subgroups of $\mathrm{SL}(n, K)$, J. Algebra 125 (1989), no. 1, 215-235. MR1012672 (91d:20051) 
[24] V. P. Platonov, Subgroups of algebraic groups over a local or global field containing a maximal torus, C. R. Acad. Sci. Paris Sér. I Math. 318 (1994), no. 10, 899-903. MR.1278148 (95a:20050)

[25] G. M. Seitz, Subgroups of finite groups of Lie type, J. Algebra 61 (1979), no. 1, 16-27. MR0554848 (81d:20039)

[26] The root subgroups for maximal tori in finite groups of Lie type, Pacific J. Math. 106 (1983), no. 1, 153-244. MR0694680 (84g:20085)

[27] N. Vavilov, Intermediate subgroups in Chevalley groups, Groups of Lie Type and their Geometries (Como, 1993), London Math. Soc. Lecture Note Ser., vol. 207, Cambridge Univ. Press, Cambridge, 1995, pp. 233-280. MR.1320525 (96c:20085)

Algebra and Geometry Department, K. L. Khetagurov North-Ossetian State University, Vatutin Street 46, Vladikavkaz 362025, Russia

E-mail address: koibaev-K1@yandex.ru

Received 18/DEC/2008

Translated by P. P. PETROV 